\title{
Vortex Formation and Stability Analysis for Shear Flows over Combined Spatially and Temporally Structured Walls
}

\author{
D.N. RIAHI* \\ Department of Theoretical and Applied Mechanics, 216 Talbot Laboratory, \\ University of Illinois at Urbana-Champaign, 104 S. Wright Street, \\ Urbana, IL 61801, USA
}

(Received 24 June 1998)

Benney's theory of evolution of disturbances in shear flows over smooth and flat boundary is extended to study for shear flows over combined spatially and temporally corrugated walls. Perturbation and multiple-scales analyses are employed for the case where both amplitude of the corrugations and the amplitude of wave motion are small. Analyses for instability of modulated mean shear flows with respect to spanwiseperiodic disturbance rolls and for the subsequent vortex formation and vortex stability are presented, and the effects of the corrugated walls on the resulting flow and vortices are determined. It is found that particular corrugated walls can originate and control the longitudinal vortices, while some other types of corrugated walls can enhance instability of such vortices.

Keywords: Shear flows; Stability analysis; Vortex stability

\section{INTRODUCTION}

In the last several decades there have been essentially two main theoretical approaches to investigate the instability of shear flows to spanwise-periodic disturbance rolls and the subsequent prediction of the observed longitudinal vortices in shear flows over smooth and

\footnotetext{
*Tel.: 217-333-0679. Fax: 217-244-5707. E-mail: d-riahi@uiuc.edu.
} 
flat walls. On one hand the work by Benney $[1,2]$ and Benney and Lin [3] was concerned with alternative theoretical approach to the threedimensional instability of parallel shear flows and was involved in interactions between first harmonics of disturbance waves and the mean shear flows. At high values of Reynolds numbers such interactions were nonlinear and took place on a fast time scale. Instability to spanwise disturbance rolls was then detected in several cases. Benney speculated that such instability may explain the well-known experimental observations of Klebanoff et al. [4]. These experiments showed clearly the evidence for spanwise-periodic longitudinal vortices maintained by the wave motion and provided proof that three-dimensional waves dominate the nonlinear flow regime. On the other hand the work by Craik [5-7], Leibovich [8] and Leibovich and Paolucci [9] considered the stability of unidirectional shear flows in the presence of small amplitude two-dimensional waves, independent of the spanwise direction, with respect to streamwise independent disturbances in the form of longitudinal vortices. The resulting instability was called later by the subsequent investigators [10] as $C L 2$, or Craik-Leibovich type2. The method of approach used in this latter work employs combination of a generalized Lagrangian-mean equations [11] plus some modeling to take into account the influence of the developing mean flow on the fluctuating part of the flow.

Both of the theoretical approaches described above intended to understand the same type of instability and its consequences on the shear flows which could explain, with more deeper physical understanding, the flow features exhibited in notable experiments such as that of Klebanoff et al. [4]. Patera and Orszag's [12] computational study of the linear instability based on infinitesimal three-dimensional disturbances superimposed on an evolved finite amplitude twodimensional shear flow led to the finding that there were some rapidly growing instabilities, and some similarities between their computational results and those of linear instability computations of Benney model carried out by Benney and Chow [13] were noticed. CL2-type instability referred to earlier in this section can also be considered as a Patera and Orszag [12] type instability for order one mean shear flows.

In the present study we apply the approach due to Benney [2] to investigate shear flow instability, vortex formation and vortex stability and control in shear flow over corrugated walls at high Reynolds 
numbers in order to obtain some estimation of the effects of surface corrugations, that can vary both in time and space, on the disturbance evolution and on the subsequent induced secondary mean flow and vortices.

\section{GOVERNING SYSTEM}

We consider an incompressible fluid flow of average depth $d$ over a wall which is corrugated with respect to both time and space variables. It is convenient to use a cartesian system of coordinated $x^{*}, y^{*}, z^{*}$, with $y^{*}=0$ being the averaged location of the wall. We nondimensionalize the governing Navier-Stokes and continuity equations by using $d, U, d / U$ and $\rho U^{2}$ as scales for length, velocity, time and pressure, respectively. Here $U$ is an appropriate velocity scale and $\rho$ is a reference (constant) density of the fluid. The non-dimensional form of these equations can then be written in the form:

$$
\begin{gathered}
\left(\frac{\partial}{\partial t}+\boldsymbol{u} \cdot \nabla\right) \boldsymbol{u}=-\nabla p+\frac{1}{R} \nabla^{2} \boldsymbol{u}, \\
\nabla \cdot \boldsymbol{u}=0 .
\end{gathered}
$$

Here $\boldsymbol{u}$ is the velocity vector $(u, v, w), p$ is the pressure, $t$ is the time variable and $R=d U / \nu$ is the Reynolds number, where $\nu$ is the kinematic viscosity.

The boundary conditions for (1.1)-(1.2) are:

$$
\begin{gathered}
\left(\boldsymbol{u}-\boldsymbol{u}_{s}\right)=-\sum_{m=1} \frac{(\delta \tilde{h})^{n}}{m !} \frac{\partial^{m}}{\partial y^{m}}\left(\boldsymbol{u}-\boldsymbol{u}_{s}\right) \quad \text { at } y=0, \\
\boldsymbol{u}=0, \quad \text { at } y=1,
\end{gathered}
$$

where $\delta$ is the magnitude of the corrugations, which is assumed to be small $(\delta \ll 1), \tilde{h}$ is corrugated wall shape function, $x=x^{*} / d$ is the streamwise variable, $y=y^{*} / d$ is the transverse variable, $z=z^{*} / d$ is the 
spanwise variable and $u_{s}=\left(u_{s}, v_{s}, w_{s}\right)$ is the velocity vector of the corrugated wall. The conditions (2.1)-(2.2) are based on the consideration that the velocity vector assumes the corrugated wall velocity vector on the corrugated wall. The terms in the right-hand-side of (2.1) arise simply by the contributions of the higher order terms in a Taylorseries expansion about $y=0$ of $\left(\boldsymbol{u}-\boldsymbol{u}_{s}\right)$.

We now apply a mean flow-first harmonic interaction type theory [2] for (1.1)-(1.2) and (2.1)-(2.2). The modification of the mean flow by the first harmonic of the superimposed waves makes the subsequent stability analysis more involved since the interactions between the mean flow and the first harmonic arise nonlinearly. Following Benney [2], we consider the following expansions for the dependent variables of the present problem:

$$
\begin{aligned}
(u, v, w, p)= & \left(u_{0}, 0,0-L x / R\right)+\delta\left(\tilde{u}_{1}, v_{0}+\tilde{v}_{1}, w_{0}+\tilde{w}_{1}, \tilde{p}_{1}\right) \\
& +\delta^{2}\left(\tilde{u}_{2}, \tilde{v}_{2}, \tilde{w}_{2}, p_{0}+\tilde{p}_{2}\right)+C . C .+\cdots,
\end{aligned}
$$

where $-L x / R$ is a linear function of $x$ providing a constant pressure gradient driving an initial classical parabolic basic flow velocity profile in the streamwise direction, the coefficients with subscript zero "0" are functions of $y, z$ and of slow variables $x_{s}$ and $t_{s}$ defined by

$$
x_{s}=\delta x, \quad t_{s}=\delta t,
$$

and the other coefficients with subscripts $n(n \neq 0)$ are functions of $\left(x, x_{s}, y, z, t, t_{s}\right)$. We shall assume that

$$
\begin{aligned}
\boldsymbol{u}_{s} & =\left(u_{s}, v_{s}, w_{s}\right) \\
& =\left(u_{s 0}, 0,0\right)+\delta\left[\tilde{u}_{s 1}+\delta^{\prime} \tilde{u}_{s 1}^{\prime}, v_{s 0}+\tilde{v}_{s 1}+\delta^{\prime} \tilde{v}_{s 1}^{\prime}, w_{s 0}+\tilde{w}_{s 1}+\delta^{\prime} \tilde{w}_{s 1}^{\prime}\right],
\end{aligned}
$$

where $\delta^{\prime}$ is another small parameter $\left(\delta \ll \delta^{\prime} \ll 1\right)$, the coefficient $u_{s 0}$ is a constant and particular forms of the other coefficients introduced in (3.3) will be specified later, but the unprimed ones are, in general, functions of $x, x_{s}, z, t$ and $t_{s}$, and primed ones are, in general, functions of $x_{s}, z$ and $t_{s}$. The surface corrugation shape function $\tilde{h}$ is likewise assumed to be of the form:

$$
\tilde{h}=h\left(x, x_{s}, z, t, t_{s}\right)+\delta^{\prime} h^{\prime}\left(x_{s}, z, t_{s}\right),
$$

where the coefficients $h$ and $h^{\prime}$ will be specified below. 
In the spirit of the mean flow-first harmonic interaction theory [2], we assume the following simple forms for the given coefficients $h, h^{\prime}$, $\tilde{u}_{s 1}, \tilde{u}_{s 1}^{\prime}, \tilde{w}_{s 1}$ and $\tilde{w}_{s 1}^{\prime}$ :

$$
\begin{aligned}
h\left(x, x_{s}, z, t, t_{s}\right) & =A\left(x_{s}, z, t_{x}\right) \exp (\mathrm{i} \alpha x-\mathrm{i} \omega t)+C . C ., \\
h^{\prime}\left(x_{s}, z, t_{s}\right) & =A^{\prime} \cos (\beta z) \exp \left(\mathrm{i} \alpha^{\prime} x_{s}-\mathrm{i} \omega^{\prime} t_{s}\right)+C . C ., \\
\tilde{u}_{s 1}\left(x, x_{s}, z, t, t_{s}\right) & =u_{s 1}\left(x_{s}, z, t_{s}\right) \exp (\mathrm{i} \alpha x-\mathrm{i} \omega t)+C . C ., \\
\tilde{u}_{s 1}^{\prime}\left(x_{s}, z, t_{s}\right) & =u_{s 1}^{\prime} \cos (\beta z) \exp \left(\mathrm{i} \alpha^{\prime} x_{s}-\mathrm{i} \omega^{\prime} t_{s}\right)+C . C ., \\
\tilde{v}_{s 1}\left(x, x_{s}, z, t, t_{s}\right) & =v_{s 1}\left(x_{s}, z, t_{s}\right) \exp (\mathrm{i} \alpha x-\mathrm{i} \omega t)+C . C ., \\
\tilde{v}_{s 1}^{\prime}\left(x_{s}, z, t_{s}\right) & =v_{s 1}^{\prime} \cos (\beta z) \exp \left(\mathrm{i} \alpha^{\prime} x_{s}-\mathrm{i} \omega^{\prime} t_{s}\right)+C . C ., \\
\tilde{w}_{s 1}\left(x, x_{s}, z, t, t_{s}\right) & =w_{s 1}\left(x_{s}, z, t_{s}\right) \exp (\mathrm{i} \alpha x-\mathrm{i} \omega t)+C . C ., \\
\tilde{w}_{s 1}^{\prime}\left(x_{s}, z, t_{s}\right) & =w_{s 1}^{\prime} \sin (\beta z) \exp \left(\mathrm{i} \alpha^{\prime} x_{s}-\mathrm{i} \omega^{\prime} t_{s}\right)+C . C .,
\end{aligned}
$$

where $\mathrm{i}=\sqrt{-1}, \alpha$ and $\alpha^{\prime}$ are streamwise wave numbers of the surface corrugation, $\beta$ is spanwise wave number of the surface corrugation, $\omega$ and $\omega^{\prime}$ are frequencies of the corrugations and C.C. indicates complex conjugate. More general forms of the above coefficient will be discussed later.

Using (3.1)-(3.4) and (4.1)-(4.6) in (1.1)-(1.2) and (2.1)-(2.2), we find that, to zeroth order in $\delta^{\prime}$, the following assumptions should be made:

$$
\left(\tilde{u}_{1}, \tilde{v}_{1}, \tilde{w}_{1}, \tilde{p}_{1}\right)=\left(u_{1}, v_{1}, w_{1}, p_{1}\right) \exp (\mathrm{i} \alpha x-\mathrm{i} \omega t)
$$

where $\left(u_{1}, v_{1}, w_{1}, p_{1}\right)$ are functions of only $x_{s}, y, z$ and $t_{s}$. Using these results in (1.1)-(1.2) and (2.1)-(2.2), we obtain the following basic flow system for the zeroth and first harmonic coefficients:

$$
\begin{gathered}
\tilde{\nabla} \cdot \boldsymbol{u}_{0}=0, \\
\frac{\partial u_{0}}{\partial t_{s}}+\tilde{\nabla} \cdot\left(\boldsymbol{u}_{0} u_{0}\right)=\frac{L}{R_{0}}+\frac{1}{R_{0}} \Delta_{2} u_{0},
\end{gathered}
$$




$$
\frac{\partial v_{0}}{\partial t_{s}}+\tilde{\nabla} \cdot\left(\boldsymbol{u}_{0} v_{0}\right)+\frac{\partial}{\partial y}\left(2 v_{1} v_{1}^{*}\right)+\frac{\partial}{\partial z}\left(v_{1} w_{1}^{*}+v_{1}^{*} w_{1}\right)=-\frac{\partial p_{0}}{\partial y}+\frac{1}{R_{0}} \Delta_{2} v_{0}
$$

$\frac{\partial w_{0}}{\partial t_{s}}+\tilde{\nabla} \cdot\left(\boldsymbol{u}_{0} w_{0}\right)+\frac{\partial}{\partial y}\left(v_{1} w_{1}^{*}+v_{1}^{*} w_{1}\right)+\frac{\partial}{\partial z}\left(2 w_{1} w_{1}^{*}\right)=-\frac{\partial p_{0}}{\partial z}+\frac{1}{R_{0}} \Delta_{2} w_{0}$

$$
\mathrm{i} \alpha u_{1}+\frac{\partial v_{1}}{\partial y}+\frac{\partial w_{1}}{\partial z}=0
$$

$$
\mathrm{i}\left(\alpha u_{0}-\omega\right) u_{1}+\frac{\partial u_{0}}{\partial y} v_{1}+\frac{\partial u_{0}}{\partial z} w_{1}=-\mathrm{i} \alpha p_{1}+\frac{1}{R}\left(\Delta_{2}-\alpha^{2}\right) u_{1}
$$

$$
\mathrm{i}\left(\alpha u_{0}-\omega\right) v_{1}=-\frac{\partial p_{1}}{\partial y}+\frac{1}{R}\left(\Delta_{2}-\alpha^{2}\right) v_{1}
$$

$$
\mathrm{i}\left(\alpha u_{0}-\omega\right) w_{1}=-\frac{\partial p_{1}}{\partial z}+\frac{1}{R}\left(\Delta_{2}-\alpha^{2}\right) w_{1}
$$

where

$$
\tilde{\nabla} \equiv\left(\frac{\partial}{\partial x_{s}}, \frac{\partial}{\partial y}, \frac{\partial}{\partial z}\right), \quad \Delta_{2}=\frac{\partial^{2}}{\partial y^{2}}+\frac{\partial^{2}}{\partial z^{2}}
$$

$R_{0}=\delta R$ is reduced Reynolds number assumed to be of order one, $\boldsymbol{u}_{0}=$ $\left(u_{0}, v_{0}, w_{0}\right)$ and "asterisk" indicates complex conjugate. These equations are subjected to the following boundary conditions:

$$
\boldsymbol{u}_{0}=\left(u_{s 0}, v_{s 0}, w_{s 0}\right), \quad\left(u_{1}, v_{1}, w_{1}\right)=\left(-A \frac{\partial u_{0}}{\partial y}, v_{s 1}, w_{s 1}\right) \quad \text { at } y=0
$$

$$
\boldsymbol{u}_{0}=\left(u_{1}, v_{1}, w_{1}\right)=0 \quad \text { at } y=1
$$




\section{DISTURBANCE SYSTEM}

The terms containing $\delta^{\prime}$ parameter in (3.3)-(3.4) and their particular form, given in (4.2), (4.4) and (4.6), imply small perturbations of (6.1)-(6.8) and (7.1)-(7.2) whose particular solution in the absence of such perturbations can be the following ones:

$$
\begin{gathered}
u_{0}=\bar{u}_{0}(y), \quad v_{0}=0, \quad w_{0}=0, \quad p_{0}=\bar{p}_{0}(y)=-2\left|v_{1}\right|^{2}, \\
u_{1}=\bar{u}_{1}(y), \quad v_{1}=\bar{v}_{1}(y), \quad w_{1}=0, p_{1}=\bar{p}_{1}(y),
\end{gathered}
$$

provided

$$
v_{s 0}=v_{s 1}=w_{s 0}=w_{s 1}=0
$$

and the following system is satisfied:

$$
\begin{gathered}
\mathrm{i} \alpha \bar{u}_{1}+\frac{\mathrm{d} \bar{v}_{1}}{\mathrm{~d} y}=0, \\
\mathrm{i}\left(\alpha \bar{u}_{0}-\omega\right) \bar{u}_{1}+\frac{\mathrm{d} \bar{u}_{0}}{\mathrm{~d} y} \bar{v}_{1}=-\mathrm{i} \alpha \bar{p}_{1}+\frac{1}{R}\left(\frac{\mathrm{d}^{2}}{\mathrm{~d} y^{2}}-\alpha^{2}\right) \bar{u}_{1}, \\
\mathrm{i}\left(\alpha \bar{u}_{0}-\omega\right) \bar{v}_{1}=-\frac{\mathrm{d} \bar{p}_{1}}{\mathrm{~d} y}+\frac{1}{R}\left(\frac{\mathrm{d}^{2}}{\mathrm{~d} y^{2}}-\alpha^{2}\right) \bar{v}_{1}, \\
\left(\bar{u}, \bar{v}, \bar{w}_{1}\right)=\left(-A \frac{\mathrm{d} \bar{u}_{0}}{\mathrm{~d} y}, 0,0\right) \quad \text { at } y=0, \\
\left(\bar{u}_{1}, \bar{v}_{1}, \bar{w}_{1}\right)=0 \quad \text { at } y=1 .
\end{gathered}
$$

Assuming the solution of the form (8.1)-(8.2) for (6.1)-(6.8) and (7.1)-(7.2), then the system for small disturbances of amplitude $\delta^{\prime}$ acting on such basic solution to be given below is satisfied by the 
disturbances' variables $\left(\tilde{\boldsymbol{u}}_{0}^{\prime}, \tilde{p}_{0}^{\prime}\right)$ and $\left(\tilde{\boldsymbol{u}}_{1}^{\prime}, \tilde{p}_{1}^{\prime}\right)$ of the form:

$$
\begin{aligned}
&\left(\tilde{u}_{0}^{\prime}, \tilde{v}_{0}^{\prime}, \tilde{p}_{0}^{\prime}, \tilde{u}_{1}^{\prime}, \tilde{v}_{1}^{\prime}, \tilde{p}_{1}^{\prime}\right)= {\left[u_{0}^{\prime}(y), v_{0}^{\prime}(y), p_{0}^{\prime}(y), u_{1}^{\prime}(y), v_{1}^{\prime}(y), p_{1}^{\prime}(y)\right] } \\
& \times \cos (\beta z) \cdot \exp \left(\mathrm{i} \alpha^{\prime} x_{s}-\mathrm{i} \omega^{\prime} t_{s}\right), \\
&\left(\tilde{w}_{0}^{\prime}, \tilde{w}_{1}^{\prime}\right)=\left[w_{0}^{\prime}(y), w_{1}^{\prime}(y)\right] \sin (\beta z) \exp \left(\mathrm{i} \alpha^{\prime} x_{s}-\mathrm{i} \omega^{\prime} t_{s}\right) .
\end{aligned}
$$

The system for the $y$-dependent coefficients of the disturbance variables is given by

$$
\begin{gathered}
\mathrm{i} \alpha^{\prime} u_{0}^{\prime}+\frac{\mathrm{d} v_{0}^{\prime}}{\mathrm{d} y}+\beta w_{0}^{\prime}=0 \\
\mathrm{i}\left(\alpha^{\prime} \bar{u}_{0}-\omega^{\prime}\right) u_{0}^{\prime}+\frac{\mathrm{d} \bar{u}_{0}}{\mathrm{~d} y} v_{0}^{\prime}=\frac{1}{R_{0}}\left(\frac{\mathrm{d}^{2}}{\mathrm{~d} y^{2}}-\beta^{2}\right) u_{0}^{\prime} \\
\mathrm{i}\left(\alpha^{\prime} \bar{u}_{0}-\omega^{\prime}\right) v_{0}^{\prime}+2 \frac{\mathrm{d}}{\mathrm{d} y}\left(\bar{v}_{1} v_{1}^{\prime *}+\bar{v}_{1}^{*} v_{1}^{\prime}\right)+\beta\left(\bar{v} w_{1}^{\prime *}+\bar{v}_{1}^{*} w_{1}^{\prime}\right) \\
=-\frac{\mathrm{d} p_{0}^{\prime}}{\mathrm{d} y}+\frac{1}{R_{0}}\left(\frac{\mathrm{d}^{2}}{\mathrm{~d} y^{2}}-\beta^{2}\right) v_{0}^{\prime} \\
\mathrm{i}\left(\alpha^{\prime} \bar{u}_{0}-\omega^{\prime}\right) w_{0}^{\prime}+\frac{\mathrm{d}}{\mathrm{d} y}\left(\bar{v}_{1} w_{1}^{\prime *}+\bar{v}_{1}^{*} w_{1}^{\prime}\right)=\beta p_{0}^{\prime}+\frac{1}{R_{0}}\left(\frac{\mathrm{d}^{2}}{\mathrm{~d} y^{2}}-\beta^{2}\right) w_{0}^{\prime} \\
\mathrm{i} \alpha u_{1}^{\prime}+\frac{\mathrm{d} v_{1}^{\prime}}{\mathrm{d} y}+\beta w_{1}^{\prime}=0 \\
\mathrm{i}\left(\alpha \bar{u}_{0}-\omega\right) u_{1}^{\prime}-\frac{\mathrm{d} \bar{v}_{1}}{\mathrm{~d} y} u_{0}^{\prime}+\bar{v}_{1} \frac{\mathrm{d} u_{0}^{\prime}}{\mathrm{d} y}+\frac{\mathrm{d} \bar{u}_{0}}{\mathrm{~d} y} v_{1}^{\prime} \\
=-\mathrm{i} \alpha p_{1}^{\prime}+\frac{1}{R}\left(\frac{\mathrm{d}^{2}}{\mathrm{~d} y^{2}}-\alpha^{2}-\beta^{2}\right) u_{1}^{\prime}
\end{gathered}
$$




$$
\begin{gathered}
\mathrm{i}\left(\alpha \bar{u}_{0}-\omega\right) v_{1}^{\prime}+\mathrm{i} \alpha \bar{v}_{1} u_{0}^{\prime}=-\frac{\mathrm{d} p_{1}^{\prime}}{\mathrm{d} y}+\frac{1}{R}\left(\frac{\mathrm{d}^{2}}{\mathrm{~d} y^{2}}-\alpha^{2}-\beta^{2}\right) v_{1}^{\prime} \\
\mathrm{i}\left(\alpha \bar{u}_{0}-\omega\right) w_{1}^{\prime}=\beta p_{1}^{\prime}+\frac{1}{R}\left(\frac{\mathrm{d}^{2}}{\mathrm{~d} y^{2}}-\alpha^{2}-\beta^{2}\right) w_{1}^{\prime} \\
\left(u_{0}^{\prime}, v_{0}^{\prime}, w_{0}^{\prime}\right)=0, \quad\left(u_{1}^{\prime}, v_{1}^{\prime}, w_{1}^{\prime}\right)=\left(u_{s 1}^{\prime}+A^{\prime} \frac{\mathrm{d} \bar{u}_{0}}{\mathrm{~d} y}, v_{s 1}^{\prime}, w_{s 1}^{\prime}\right) \text { at } y=0 \\
\left(u_{0}^{\prime}, v_{0}^{\prime}, w_{0}^{\prime}\right)=0, \quad\left(u_{1}^{\prime}, v_{1}^{\prime}, w_{1}^{\prime}\right)=0 \quad \text { at } y=1
\end{gathered}
$$

\section{EXAMPLE}

In this section we explore some aspects of the analytical results for the inviscid flow case of the theory presented in the previous two sections when it is applied to a simple shear flow with a free surface. Such simple shear flow is a particular extension of the problem studied by Benney and Chow [13], of a shear flow over a flat and smooth boundary, to the case for the flow over a moving corrugated boundary.

Following [13], we assume $R=\infty$ and the following linear profile

$$
\bar{u}_{0}(y)=F y,
$$

where $F$ is a constant. In addition, boundary conditions in the absence of perturbations, described in the previous section, are now [13]

$$
\begin{gathered}
\bar{v}_{0}=\frac{\mathrm{d} \bar{p}_{1}}{\mathrm{~d} y}=0 \quad \text { at } y=0 \\
\bar{v}_{0}=\frac{\mathrm{d} \bar{p}_{1}}{\mathrm{~d} y}-\left(\alpha \bar{u}_{0}-\omega\right)^{2} \bar{p}_{1}=0 \quad \text { at } y=1 .
\end{gathered}
$$


Using (11) in (8.4)-(8.6) and eliminating $\bar{u}_{1}$ and $\bar{v}_{1}$, we find

$$
\frac{\mathrm{d}^{2} \bar{p}_{1}}{\mathrm{~d} y^{2}}-\frac{2 \alpha F}{\left(\alpha \bar{u}_{0}-\omega\right)} \frac{\mathrm{d} \bar{p}_{1}}{\mathrm{~d} y}-\alpha^{2} \bar{p}_{1}=0 .
$$

The system (12)-(13) for $\bar{p}_{1}$ represents an eigenvalue problem for $\omega$. Similar to the consideration in [13], critical layers will not be considered here, and using transformation of the form:

$$
G(y)=-\frac{1}{(\alpha F y-w)} \frac{\mathrm{d} \bar{p}_{1}}{\mathrm{~d} y},
$$

eigenfunction is found to be of the form:

$$
\bar{p}_{1}=\mathrm{i} B\left[\left(\frac{\omega}{\alpha}-F y\right) \cosh \alpha y-\frac{F}{\alpha} \sinh \alpha y\right],
$$

where $B$ is a constant. Using (15) in (12.2) lead to the eigenvalues given by

$\omega=\left(\frac{\alpha}{2}\right)\left\{(2 F-F \tanh \alpha / \alpha) \pm\left[(F \tanh \alpha / \alpha)^{2}+4 \tanh \alpha / \alpha\right]^{1 / 2}\right\}$.

Let us now consider the case where

$$
\alpha=0 .
$$

Using (17) in (8.4)-(8.6) and applying zero boundary conditions for $\bar{v}_{1}$ and (12.1)-(12.2), we find

$$
\bar{u}_{1}=\bar{v}_{1}=\bar{p}_{1}=0,
$$

provided

$$
\omega \neq 0
$$

Applying the instability system (10) for the present example, we find that three-dimensional instability of the basic flow follows, for a wide 
range of values for $\alpha^{\prime}$ and $\omega^{\prime}$ [13], provided (17) and (19) are not satisfied and if the disturbance mode is not due to the corrugated wall. If the disturbance mode is due to the corrugated wall, then the basic flow is stable for real values of $\omega^{\prime}$. However, if the conditions (17) and (19) are satisfied, for particular corrugated walls, then the basic flow remains stable and no three-dimensional instability takes place regardless of the type of the disturbance mode, provided $\omega^{\prime}$ for the corrugated wall is real as is usually assumed. In addition, for the case where (17) and (19) are not satisfied, no three-dimensional instability occurs regardless of the type of the disturbance mode, provided

$$
\beta=0 .
$$

\section{SOME CONCLUSIONS}

(i) Certain structured walls can prevent shear flow destabilization by three-dimensional disturbances.

(ii) Structured walls can provide neutral flow disturbances leading to a modified form of the basic shear flows.

(iii) Vortex formation in the shear flows can be prevented if the flow catalyst, such as, for example, two-dimensional waves superimposed on a one-dimensional original mean shear flow, can be suppressed by the presence of some types of structured boundary.

(iv) Three-dimensional instabilities of slightly perturbed twodimensional spatially periodic shear flows can take place in the presence of corrugated walls if these walls have particular amplitude and shape that reinforce the two-dimensional spatial periodicity of the shear flows.

(v) Stabilizing effects of the corrugated walls can be much more stronger if the amplitude of their non-periodic component be comparable to that of the waves which are resonant with the mean flow.

\section{References}

[1] D.J. Benney, A nonlinear theory for oscillation in a parallel flow. J. Fluid Mech. 10, 209-236 (1961). 
[2] D.J. Benney, The evolution of disturbances in shear flows at high Reynolds numbers. Stud. Appl. Math. 70, 1-19 (1984).

[3] D.J. Benney and C.C. Lin, On the secondary motion induced by oscillations in a shear flow. Phys. Fluids 3, 656-657 (1960).

[4] P.S. Klebanoff, K.D. Tidstrom and L.M. Sargent, The three-dimensional nature of boundary-layer instability. J. Fluid Mech. 12, 1-34 (1962).

[5] A.D.D. Craik, The generation of Langmuir circulation by an instability mechanism. J. Fluid Mech. 81, 209-223 (1977).

[6] A.D.D. Craik, Wave-induced longitudinal-vortex instability in shear flows. J. Fluid Mech. 125, 37-52 (1982).

[7] A.D.D. Craik, Wave Interactions and Fluid Flows. Cambridge University Press, Cambridge (UK) (1985).

[8] S. Leibovich, Convective instability of stably stratified water in the ocean. J. Fluid Mech. 82, 561-585 (1977).

[9] S. Leibovich and S. Paolucci, The instability of the ocean to Langmuir circulation. J. Fluid Mech. 102, 141-167 (1981).

[10] W.R.C. Phillips and $\mathrm{Z}$. Wu, On the instability of wave-catalysed longitudinal vortices in strong shear. J. Fluid Mech. 272, 235-254 (1994).

[11] D.G. Andrews and M.E. McIntyre, An exact theory of nonlinear waves on a Lagrangian mean flow. J. Fluid Mech. 89, 609-646 (1978).

[12] A.T. Patera and S.A. Orszag, Transition and turbulence in planar channel flows. In Proc. 7th Internat. Conf. on Numerical Methods in Fluid Dynamics (Eds. R.W. MacCormack and W.C. Reynolds), Springer, Berlin, p. 329 (1980).

[13] D.J. Benney and K. Chow, Instabilities of waves on a free surface. Stud. Appl. Math. 74, 227-243 (1986). 


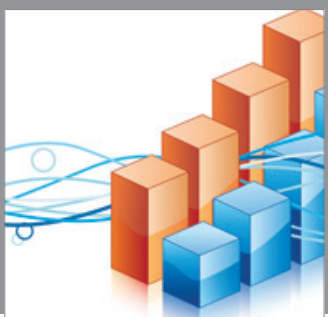

Advances in

Operations Research

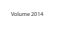

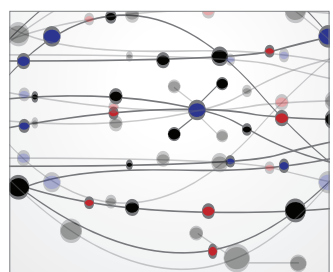

\section{The Scientific} World Journal
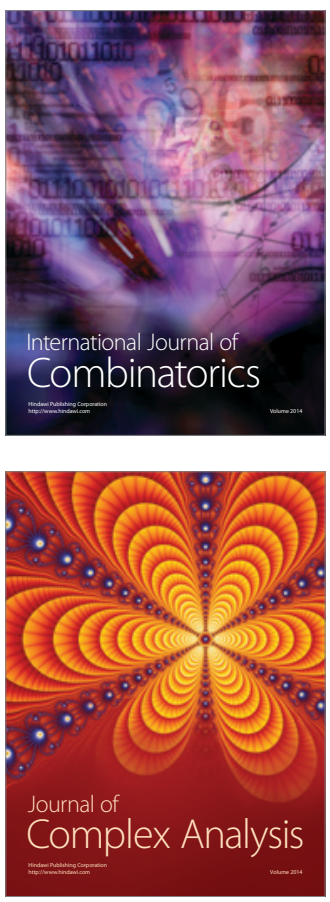

International Journal of

Mathematics and

Mathematical

Sciences
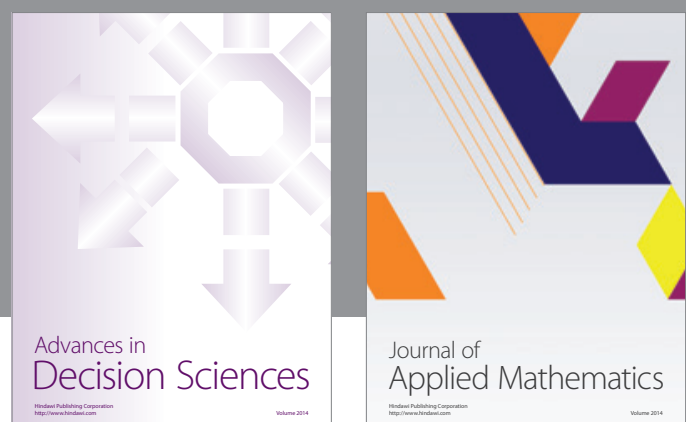

Journal of

Applied Mathematics
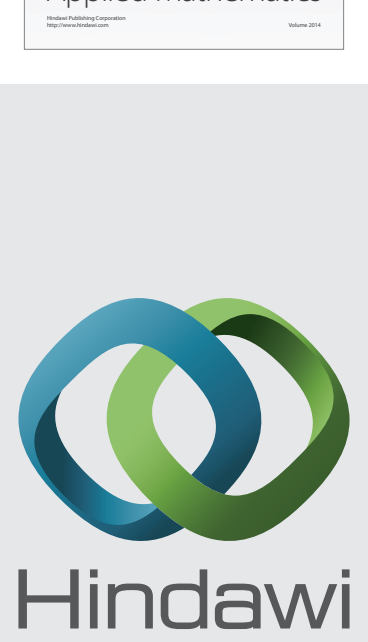

Submit your manuscripts at http://www.hindawi.com
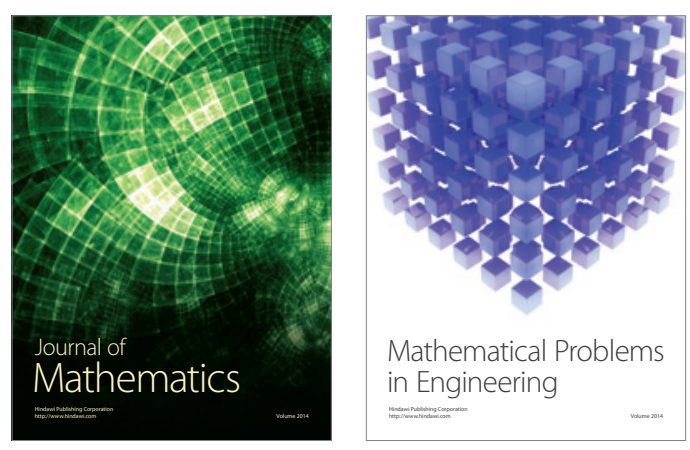

Mathematical Problems in Engineering
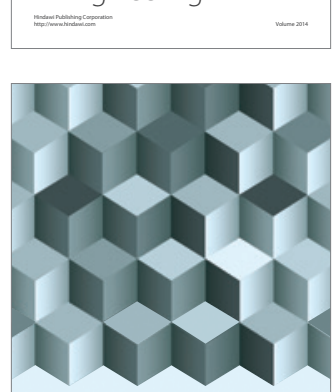

Journal of

Function Spaces
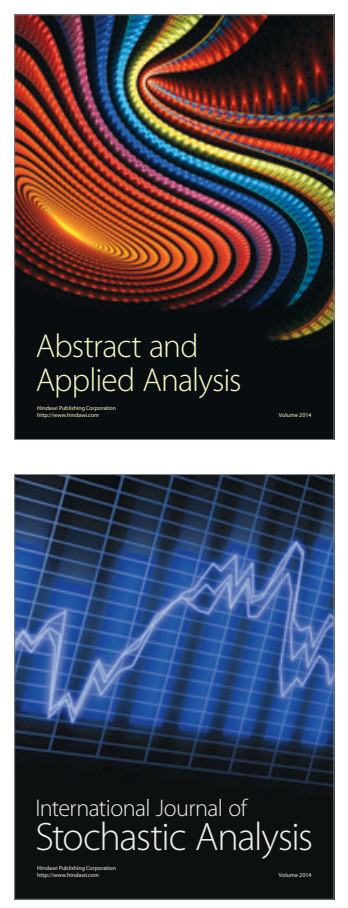

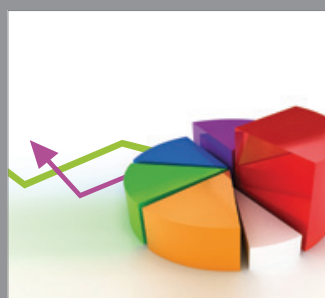

ournal of

Probability and Statistics

Promensencen
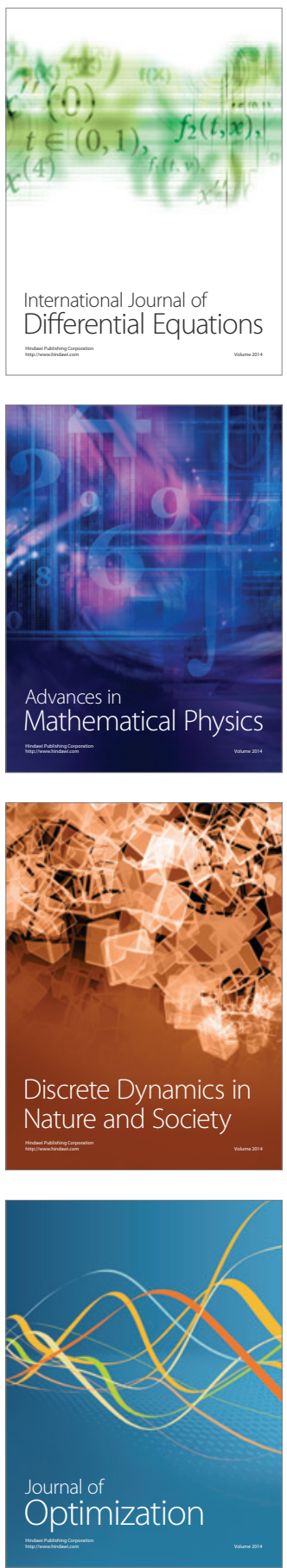